\title{
Editorial: The challenge of off-label prescribing
}

Before medications can be prescribed, pharmaceutical companies must apply for approval from their relevant government authority. Yet prescribing a medication outside of its registration (licensed) or off-label prescribing (unlicensed use) is not illegal, and common in palliative care. The practice has emerged because many symptom management guidelines are not evidence based and often include recommendations for the administering of medication outside of their registered use. As a result, prescribing off-label medications has become widespread and normalised in many palliative care services. It is estimated that up to a quarter of all prescriptions for licensed drugs in palliative care are being used for unlicensed indications or are administered by an unlicensed route (Pavis and Wilcock, 2001).

An Australian and New Zealand survey of palliative care physicians' $(n=105)$ off-label prescribing practices found the majority (89\%) were not aware of their palliative units having a policy requiring patient consent for off-label prescribing. Half (50\%) correctly identified licensed medication drugs being provided for unlicensed use (To et al, 2013). A quarter of these physicians neither documented the rationale for off-label prescribing nor obtained informed consent from patients. Most (70\%) indicated their organisation did not restrict unlicensed prescribing. Similarly, a UK study found that even fewer services had an off-label prescribing policy (2\%) (Pavis and Wilcock, 2001). This study also noted there was minimal documentation in the patient's medical records of the rationale for prescribing unlicensed medications, or evidence of written or verbal consent being obtained from the palliative care patient or their substitute decision-maker. These studies highlight the importance of palliative care services establishing policies to guide off-label prescribing.

The management of common symptoms experienced by patients with advanced illness has evolved rapidly, with several completed randomised control trials investigating the effectiveness of commonly used off-licence medications. Using ketamine as an example, a trial found that this medication, as an adjunct to opiates and co-analgesics for cancer pain, did not have a net clinical benefit. In fact, the number of patients needed to treat for one additional patient to have a positive outcome from ketamine was $25(95 \% \mathrm{Cl}$, six to $\infty)$ (Hardy et al, 2012). Regular monitoring identified that patients experienced unnecessary adverse side-effects. While these results have not been universally welcomed and need to be replicated, they raise a number of clinical and ethical considerations for palliative care nursing practice.

If we are truly committed to not causing harm, and minimising suffering and discomfort at the end of life, then we need to rethink our acceptance of administering unlicensed medications and work towards ensuring that the symptom management we provide is always grounded in the best available evidence. If we are to ensure that the care we provide is clinically, legally and ethically sound and justified, we need to be prepared to challenge the ongoing prescribing of these medications, especially where new evidence does not support their ongoing use, and seek consent from our patients or their substitute decision-maker before these medications are prescribed and administered.

Jane Phillips, Director, Centre for Cardiovascular and Chronic Care, University of Technology Sydney 
Hardy J, Quinn S, Fazekas B, Plummer J et al (2012) Randomised, double-blind, placebo-controlled study to assess the efficacy and toxicity of subcutaneous ketamine in the management of cancer pain. J Clin Oncol 30(29): 3611-7

Pavis $\mathrm{H}$, Wilcock A (2001) Pre- scribing of drugs for use out- side their licence in palliative care: survey of specialists in the UK. BMJ 323(7311): 484-5

To TH, Agar M, Shelby-James Tet al (2013) Off-label pre- scribing in palliative care-a cross-sectional national sur- vey of palliative medicine doctors. Palliat Med 27(4):320-8 